\title{
Roots into functional nodes: exploring locality and semi-lexicality
}

\begin{abstract}
We explore certain predictions of the theory first presented in Author 1 and Author 2 (2014), whereby roots correspond to (lately inserted) Vocabulary Items that phonologically and semantically interpret functional nodes. A prominent locus for the insertion of roots are categorizers $(a, n, v)$. Roots such as CAT or UP are Vocabulary Items that count with no context of insertion and are insertable into any categorizer, accounting for the categorial variability of simple words. Derivational affixes like -ation or -al also correspond to roots inserted into little head categorizers ( $n$ and $a$, respectively, in this case), but their Vocabulary Items have a context of insertion accounting for their categorial rigidity and their c-selection properties. The exploration proposed here focuses, first, on the locality properties of morphemic interactions at the phonological and semantic interface, namely, allomorphy and allosemy. We show that our approach is superior to those in which roots are distinct nodes in the syntax. Second, we suggest that the phenomenon of semi-lexicality, as illustrated by classifiers, can be modeled as the insertion of roots into inflectional nodes.
\end{abstract}

Keywords: allomorphy, allosemy, locality, semi-lexicality, roots

\section{Introduction}

In A(uthor) 1 and A(uthor) 2 (2014), our previous work on this topic, we propose a $\mathrm{R}$ (oot-)i(nto-)F(unctional node) approach to the integration of roots in the morphosyntactic structure: roots are VIs inserted at the interface directly into functional nodes. The main illustration is provided by the insertion of roots into the categorizers provided by $\mathrm{UG}-n, a, v{ }^{1}$ Thus, we maintain the standard idea that categorizers are manipulated by syntax (unlike De Belder 2011), but we treat roots as non-syntactic objects: as VIs that interpret the categorizers at the interface. One of the empirical observations on which the theory is based is the lack of crosslinguistic morphological evidence for pure categorizing elements, i.e., for elements that merely signal category and do not thus show any other conceptual dimension of meaning. Rather, the elements associated with particular categories, like -ity, -al, -ness, etc., also show conceptual meaning (De Belder 2011, Lowenstamm 2014) and a variety of exponents that is arguably not always due to allomorphy. Derivational (categorizing) morphemes behave thus differently from inflectional ones such as $\mathrm{T}_{[\mathrm{PST}]}$ or $\mathrm{Num}_{[\mathrm{PL}]}$, which count with dedicated exponents and limited allomorphy, and a fixed semantics in the different languages.

In this paper we wish to explore the RiF approach further. We briefly summarize it in Section 2. In Section 3 we deal with derivational affixes. We add empirical evidence to that presented in A1\&A2 that shows that these elements share non-trivial properties with open-class lexical items, and we present an analysis to capture this similarity, but also their differences. We dedicate section 4 to the predictions that the theory makes with respect to allomorphy (Section 4.1) and allosemy (Section 4.2). In Section 5 we hint at how the theory can also shed light on the nature of the phenomenon of semilexicality. Section 6 concludes.

\footnotetext{
${ }^{1}$ See AUTHOR $1(2010,2016)$ and Wood and Marantz (2017) for little $p$.
} 


\section{The Root-into-Functional-node approach}

Syntax does not manipulate roots. It only manipulates functional heads, among them the so-called little head categorizers: $n, v, a$. We assume that, for the derivation to converge at LF, these are the first terminals selected. To solve the linearization problem inherent to First Merge, we propose that the categorizer undergoes so-called self-merge (Guimarães 2000, Kayne 2009), yielding a maximal category. For instance, the nominalizer $n$ merges with itself, yielding the set $\{n, n\}$, which, by the Extensionality Axiom of Set Theory (Partee et al. 1993), is equivalent to the singleton $\{n\}$. This is an $n \mathrm{P}$, and it can now merge with a functional head. If we abstract away from other functional material like Gender/Class, Number, etc., this head could be D, yielding a DP:

\section{(1) $[\mathrm{DP} D[\mathrm{nP} n]]$}

Structures are transferred to the interfaces cyclically (Embick 2010, Marantz 2013) and terminals undergo Vocabulary Insertion. We assume the Subset Principle (Halle 1997), which stipulates that out of a set of VIs competing to be inserted into a given terminal provided with a set of morphosyntactic features, $S$, only the one containing the biggest subset of $S$ will be inserted.

Most roots, like that present in cat or yellow, are not specified for any feature, which we represent with the empty set:
(2) a. $\mathrm{CAT} \leftrightarrow \varnothing$
b. WALK $\leftrightarrow \varnothing$
c. SPY $\leftrightarrow \varnothing$
d. YELLOW $\leftrightarrow \varnothing$

Since the empty set is the smallest subset of any set, the Subset Principle entails that roots like those in (2) will be inserted in those terminals for which there is no minimally specified VI. Take, for instance, the terminal $n$ of (1). We take it that English does not possess any VI specified for just the feature $n .^{2}$ This opens the way for cat or any other exponent whose VI is of the format in (2) to be inserted in $n$ (we use a leftwards white arrow to represent the selection of a VI for a terminal):

\section{(3) $[\mathrm{DP} \mathrm{D} \diamond$ the $[\mathrm{nP} \mathrm{n} \hookleftarrow \mathrm{CAT} / \mathrm{WALK} / \mathrm{SPY} /$ YELLOW/...] $]$}

Importantly, since these roots have exactly the same context of insertion (none), any of them is insertable in a categorial node. This accounts for the idea that there is a free choice as far as root insertion is concerned (Marantz 1997). In Section 4 we will provide a more articulate theory of Vocabulary Items and Vocabulary Insertion able to deal with root allomorphy and allosemy in a strictly localistic and cyclical way.

It should be clear that roots are predicted, in this theory, to be insertable in any node

\footnotetext{
${ }^{2}$ We thus partly endorse Lowenstamm's (2014:232) hypothesis that "categories have no exponents". However, we believe that derivational affixes do, as we show in Section 3 (see Borer [2013], Creemers, Don and Fenger [2017]).
} 
for which there is no dedicated VI, including inflectional nodes. This latter possibility is suggested in Section 5.

\section{Open-class items and derivational affixes}

The general research agenda of splitting open-class lexical items their category has been pushed farther in a few recent works that propose that derivational affixes, prima facie possessing a category, have to be stripped of it also. Thus, De Belder (2011) develops a theory in which syntax does not operate at all with lexical categories, and where derivatives are actually also category-free roots (see also De Belder \& van Craenenbroeck [2015]). A similar line is pursued by Lowenstamm (2014). Supporting his analysis with evidence from cyclicity effects in stress assignment in derived words, this author proposes that derivatives like -ic (atomic) are roots. However, he does not eliminate the dedicated categorizing morphemes $(a, n, v)$ from the representation. Even more recently, Creemers, Don \& Fenger (2017), basing on a reexamination of the Dutch data, have introduced a nuance into this idea: they concede that derivational affixes can be roots, but they point out that some are actually grammatical heads.

Before we propose an analysis of derivatives in terms of the theory sketched in Section 2, we would like to present some empirical arguments that these elements involve roots, adding to those presented in De Belder's (2011), A1\&A2, and Lowenstamm's (2014).

First, the existence of minimal pairs of derived words of the same category sharing a root but involving different categorizers and a clear difference in meaning. This is exemplified with the next pairs of Catalan words:

a. mols-ós

moss-os

'abundant in moss'

a. ferr-ós

iron-os

'containing or abundant in iron'

a. cendr-aire

ash-air

'person who buys and sells ash' b. mols-ut

moss-ud

'(soft) like moss'

b. ferr-is

iron-iss

'strong as iron'

b. cendr-er

ash-er

'ash-tray'

It is difficult to see how theories not supporting the root-like nature of derivatives can explain contrasts like the above. Theories like Marantz (2013), Wood (2015), or Wood \& Marantz (2017) allow for contextual allosemy of categorizing functional nodes like $v$. For instance, Wood (2015: 28), proposes that $v$ has a causal alloseme when it combines with an eventuality. Crucially, the different denotations (allosemes) of the same functional head are context-dependent: a local relation with some other element in the configuration (in this case, an eventuality) triggers the retrieval of a particular alloseme of the functional head at LF. However, this type of explanation can arguably not be carried over to the cases in (4) through (6). In particular, it is difficult to posit rules for contextual allosemy for the $a$ and $n$ categorizers, since the syntactic configuration underlying the members of these pairs is the same, and thus the context in which the different suffixes are interpreted is the same. Thus, it can be shown that the members of these pairs behave alike as far as grammar is concerned. 
As for the adjectives in (4) and (5), the four of them are predicative and gradable, as shown below:

a. un tronc molt mols-ós

a trunk very moss-os

'a very mossy trunk' b. uns llavis molt mols-ut-s

some lips very moss-ud-PL

'very soft lips'

b. unes espatlles molt ferr-iss-e-s some shoulders very iron-iss-F-PL 'very robust shoulders'

Both types of adjectives can be in attributive and predicative contexts, showing that they have the same syntactic distribution:

(9) a. Té les galtes molt molsudes/molsoses [attributive]

Has the cheeks very moss-ut FEM.PL /moss-os FEM.PL

'She/he has very fleshy cheeks/ ${ }^{\#}$ mossy cheeks'

b. Li vaig veure les galtes molsudes/molsoses [predicative].

DAT AUX see the cheeks moss-ut.FEM.PL/moss-ós.FEM.PL

'I saw her cheeks very fleshy/ ${ }^{\#}$ mossy'

Analogously, both (6)a and (6)b are common, count nouns, admitting, for instance, the indefinite article $u n$ ' $a$ ' with a token interpretation: un cendraire 'an ash-seller', un cendrer 'an ash-tray'.

We believe that any allosemy approach to the semantic difference between these pairs of derivative affixes is therefore to be abandoned in favour of one in which they are actually different affixes. However, it is also a fact that -os and $-u d,-o s$ and -iss, and -air and -er share category: $a$ in the first two pairs, $n$ in the last pair. The conclusion is that the difference must be of purely conceptual nature, involving the use of different roots: OS/UD, OS/ISS, and AIR/ER. ${ }^{3}$

Another property shared by run-of-the-mill roots and derivational affixes is the fact that both are associated with purely inflectional properties in languages like Spanish

\footnotetext{
${ }^{3}$ Embick \& Marantz (2008) also mention the case of derived words which, sharing both category and root, possess different meanings. Specifically, they tackle the case of the nouns cover and coverage, and propose that these nouns involve the same underlying syntactic structure, one in which an $n$ categorizer is directly attached to the root: [nP $n$ COVER]. At Vocabulary Insertion there would be a free choice between the $\varnothing$ and the -age exponents, by virtue of the fact that the respective VIs both make reference to the root COVER. In turn, the difference in meaning between cover and coverage would be analogous to that between sofa and couch, or thief and stealer, boiling down to their occupying different semantic subspaces. We take issue with this analysis. Cover and coverage, and, in general, zero-derived vs non-zero-derived nominalizations in English are grammatically different, as has been shown in detail by Borer $(2003,2013)$. Specifically, zero-derived nominals are not complex event nominals, in Grimshaw's (1990) sense, or argument structure nominals in Borer's (2003) sense, while nominals derived with an overt affix are, as the next contrast shows: the media coverage/*cover of the refugee crisis. It is unclear how this difference may emerge from Embick \& Marantz's (2008) analysis, since the structure attributed to the nominals is the same. By contrast, the difference is naturally derived in our theory; while cover corresponds to the structure [ $\left.{ }_{n \mathrm{P}} \mathrm{n}\right]$, with COVER being inserted directly into $n$, coverage corresponds to the structure $[\mathrm{nP} \mathrm{n}[\mathrm{vP} \mathrm{v}]]$, with COVER inserted into $v$ and AGE into $n$. Only the latter possesses an eventive layer $(v)$ able to sustain argument and event structure. See Borer (2003, 2013157ff.) for much illuminating discussion and a different analysis.
} 
and other Romance languages. Thus, both roots in nouns and nominalizing suffixes are associated with the so-called word markers (Harris 1991) and gender. For instance, both the root VAS 'glass' and the nominalizer -ism '-ism' are associated with the word marker $-o$ and masculine gender. Likewise, the root CAM 'bed' and the nominalizer $-u r$ are associated with the word marker $-a$ and feminine gender (see Harris 1999):
a. el
vas-o, el
dada-ísm-o $\quad(* l a$
vas-a, etc.)
the(M)
glass- $o(\mathrm{M})$ the $(\mathrm{M})$
dada-ism- $o$ (M)the(F)
glass- $a(\mathrm{~F})$
b. la
cam- $a$, la
alt-ur-a $\quad(* e l$
cam-o, etc.)
the(M) bed- $a$.F the(F)
tall-ur-a.F the(M) bed-o.M

Similarly, both roots in verbs and also verbalizers are associated with a particular conjugation class. Thus, while roots like CANT 'sing' and verbalizers like $-i z$ '-ize' are associated with the $a$-conjugation class (cant-a- $r$ 'to sing', movil-iz-a- $r$ 'to mobilize'), roots like BEB 'drink' and verbalizers like -EC are associated with the $e$-conjugation class (beb-e-r 'to drink', flor-ec-e-r 'to bloom'). ${ }^{4}$

This evidence strengthens the hypothesis that derivational affixes involve roots. In fact, in A1 \& A2 (2014) we assumed the default hypothesis that derivational affixes should be provided with exactly the same analysis as roots like cat, i.e., an analysis where both kinds of items have VIs with no contexts of insertion. From this perspective, a derivative like -al (cf. remov-al) could be inserted into any categorial node, as per the algorithm explained in Section 2. In this paper we wish to modify this position. Derivational affixes do involve roots. As such, they count with an entry in the Encyclopaedia, i.e., their semantics is not read off a set of morphosyntactic features, as in the case of inflectional terminals. However, their VIs are equipped with a context of frame that determines both their category and their c-selection properties. We therefore reduce these two properties of derivatives to a condition on Vocabulary Insertion. Moreover, from this perspective derivatives are pretty much like idioms, in Borer's (2005) sense: pieces of phonological and conceptual information associated with grammatical information.

Consider, for instance, the two Catalan adjectivizers presented in (4) count with the following VIs:
a. OS $\leftrightarrow \mathrm{a} /[$
b. UD $\leftrightarrow \mathrm{a} /[$

These VIs ensure that OS and UD will only be insertable in $a$ nodes that embed an $n$ node. For instance, the Vocabulary Insertions involved at the PF stage of the two

\footnotetext{
${ }^{4}$ An anonymous reviewer asks why couldn't functional morphemes or their exponents have class features. Actually, we adopt Acquaviva's (2009) idea that class features are necessarily hosted in functional morphemes, and not in roots —or, in our case, root exponents. For instance, in section 4.2 we develop an analysis of gender in Spanish that assumes that this is an independent functional projection merged on top of $n$ and hosting class features of sorts ([IFEM]), which are in turn crucial in the semantic interpretation of the noun. For lack of space, we will restrict ourselves to mentioning one of Acquaviva's theoretical arguments in favour of this position: if roots were provided with class features, say conjugation class, they would indirectly be provided with a category (verb, in this case), jeopardizing one of the main tenets of Distributed Morphology. The reader is referred to Acquaviva's work for empirical arguments also.
} 
adjectives in (4) can be represented as follows: ${ }^{5}$

(12) $\quad$ [ap a

molsós 'abundant in moss' / molsut '(soft) like moss'

Since OS and UT have exactly the same context of insertion in their VIs, they are freely insertable as denominal adjectivizers. The only differences between them are their phonology and their conceptual semantics, based on their two different phonological exponents $(/ \mathrm{oz} /, / \mathrm{ud} /)$ and semes ("abundant in moss", "(soft) like moss"). ${ }^{6}$

\section{Exploring locality}

One of the major concerns in a syntactic theory of morphology is whether strict locality conditions can be defined determining morphological phenomena like multiplity of form (allomorphy) and multiplicity of meaning (allosemy). It is the purpose of this section to show how the RiF approach may shed light on this issue and may be superior to theories endorsing the idea that the root and the categorizer of a derived word are different terminals.

According to the RiF approach, the categorizer $c$ and the root do not correspond to two different terminal nodes. This is shown in (13)a. Rather, the root is not a node, but a VI inserted in $c$. The scenario in (13)b, predicted by an approach in which roots are syntactic objects, is different, since the root and $c$ correspond to different terminal nodes:

(13) a. [FP F [cP $\mathrm{c} \Leftarrow$ ROOT] $]$

b. [FP F [

Since, as standardly assumed (Embick [2010], Marantz [2013]), the adjacency between the trigger and the target is a prominent locality condition regulating allomorphic and allosemic effects, it is clear that the theories generating (13)a and (13)b make different predictions as to whether $\mathrm{F}$ and ROOT may engage in allomorphic/allosemic interactions. In particular, assuming (13)a, this is straightforwardly possible. Assuming (13)b, it is not, unless some extra mechanism is adopted.

In order to account for allomorphy and allosemy in our framework we have to first make precise our theory of how the syntactic information is interpreted by the interfaces. At the point at which the output of the syntactic derivation is transferred, Vocabulary Selection, i.e., the selection of Vocabulary Items to be used in the interpretation of terminal nodes, take place. Vocabulary Selection applies in a bottomup fashion, going from the more embedded to the less embedded terminals (cf.

\footnotetext{
${ }^{5}$ We are also abstracting away from the final linear order relations between the morphemes (mols-ós, mols-ut).

${ }^{6}$ An anonymous reviewer asks whether MOLS could be directly inserted into $a$, generating an adjective on its own that could coexist with molsut and molsós. We believe that this option is possible, as we can use mols $a$ as an adjective with even degree modifiers like molt 'very':

(i) Un color molt molsa.

a colour very moss

'A colour very much like that of moss.'
} 
We take a VI to be composed of, on the one hand, a set containing phonological $(\Phi)$ and semantic information $(\Sigma)$ to be used by each interface, and, on the other hand, a context of insertion, $\mathrm{C}$ :

$$
\{\Phi, \Sigma\} \leftrightarrow C
$$

VIs enter into a competition for insertion, regulated through the Subset Principle (see Section 2). $\Phi$ and $\Sigma$ are themselves sets of phonological exponents, $\varphi$, and semes, $\sigma$, respectively:

$$
\begin{aligned}
& \text { a. } \Phi=\left\{\varphi_{1}, \varphi_{2}, \ldots\right\} \\
& \text { b. } \Sigma=\left\{\sigma_{1}, \sigma_{2}, \ldots\right\}
\end{aligned}
$$

Upon selection of a VI for a given terminal, respectively, $\Phi$ and $\Sigma$ are inspected in order to interpret the terminal node through one exponent and one seme: Exponent Insertion and Seme Insertion, respectively. A VI involves allomorphy if its $\Phi$ set contains more than one element, and it involves allosemy if its $\Sigma$ set contains more than one element. The elements contained in $\Phi$ and $\Sigma$ are selected according to the Elsewhere Principle: the most specified ones are used first in either Exponent or Seme Insertion, where specification is measured in terms of reference to contextual information.

We will deal with allomorphy in Section 3.1, and with allosemy in Section 3.2. However, we would like to provide first some remarks on how the approach works with respect to the interaction between the (most embedded) root and subsequent categorizers, as in break-abil-ity or remov-al

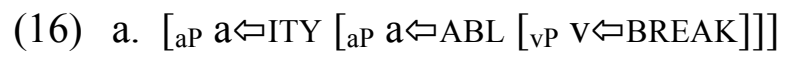

b. [nP $\mathrm{n} \diamond \mathrm{AL}[\mathrm{vP} \mathrm{V} \prec \mathrm{REMOVE}]$

We follow Embick's (2010) proposal of cyclic Spell-Out: when a new categorizing head $y$ is added to the derivation, any cyclic domain headed by cyclic $x$ in its complement is spelled out. Insertion into $x$ cannot be determined by $y$, but insertion into $y$ may be determined by the choice for $x$. In the case of breakability, the syntax yields first the singleton [vP V], which is spelled out when the higher node $a$ is merged.

\footnotetext{
${ }^{7}$ Although we are not endorsing spanning or phrasal Spell-Out, as do Bye and Svenonius's (2012), our Vocabulary Selection feeds both interfaces, and so it is similar to their L-matching stage. In a theory assuming strict Late Insertion, such as ours (or theirs), providing a common access point to both interfaces ensures the preservation of the Saussurean coupling of form and meaning. We note, importantly, that the assumption of a common access point, on the one hand, and, that of a bipartite structure for VIs (including phonological and conceptual information), is not more onerous to the grammar than other architectures assumed in Distributed Morphology. For instance, in the seminal work of Marantz (1997) the assumption was made that the Encyclopaedia somehow has access to the choices made at Vocabulary Insertion, creating a link between PF and the semantic interface and thus making the architecture more complex. Similarly, the theories in which roots are early inserted as indices whose phonological and conceptual interpretation is retrieved at each of the interfaces, like Harley (2014), just distribute in different items - the index used in syntax, the VI at PF, and the Encyclopaedia Entry at the semantic interface - what we are suggesting comes together in the same package: the VI.
} 
The root BREAK is inserted into $v$, and $a$ is not present in this cycle. In turn, $a$ is spelled out when the higher $n$ node is added. Insertion into $a$ could be in principle be determined by the choice made in the previous cycle (BREAK), but this is not the case with ABLE. Finally, $n$ is spelled out and ITY is inserted in it, in this case, as determined by the previous choice for a, namely, ABLE.

The case of removal works similarly. Upon merger of $n$, the cycle headed by $v$ is spelled out, with insertion of REMOVE. The head $n$ is not present in this cycle. However, the choice for $n(\mathrm{AL})$ may be determined — as is the case - by the previous choice for $v$ (REMOVE).

\subsection{Locally determined root allomorphy without Readjustment Rules}

According to Embick (2010) or Marantz (2013), allomorphic interactions are restricted by two locality conditions. First, the trigger and the target must be within the same Spell-Out domain, i.e., the portion of the structure being inspected by PF. Second, they must be adjacent to each other.

Embick's (2010) theory makes explicit that the stretch of structure represented in (13) pertains to the same Spell-Out domain, since only a categorizing head is present. According to (13)a, the choice of exponent at the $c$ node can be determined by the morphosyntactic features of F. In other words, F may determine an allomorph of the root. For instance, and assuming that in a past tense form in English the tense morpheme $\mathrm{T}_{[\mathrm{PST}]}$ is directly above the categorizer, $\mathrm{T}_{[\mathrm{PST}]}$ is predicted to be able to trigger allomorphy in the verbal root, since the verbal root boils down to a VI inserted in $\mathrm{v}$, which is adjacent to $\mathrm{T}_{\text {[PST] }}$. Let us apply this idea to the derivation of a form like caught. Syntax yields the following configuration to PF:

$$
\text { a. }\left[\mathrm{TTP}_{[\mathrm{PST}]}[\mathrm{vP} \mathrm{V}]\right]
$$

At the point of transfer to the interfaces, Vocabulary Selection takes place, from the more embedded terminal to the less embedded one, i.e., it starts at $v$. The VI corresponding to the root $\mathrm{CATCH}$, shown below, relates the sets $\Phi$ and $\Sigma$ to the empty set. Since we are dealing with allomorphy here, we abbreviate the set $\Sigma$ as $\Sigma_{\text {САтсH. We }}$ use the written form of the allomorphs, rather than the phonological representation:

$$
\left\{\left\{\text { caugh/ } \mathrm{T}_{[\mathrm{PST}]}[\mathrm{]}], \text { catch }\right\}, \Sigma_{\mathrm{CATCH}}\right\} \leftrightarrow \varnothing
$$

Since the empty set is a subset of all sets, this VI may be selected to interpret the $v$ node phonologically and semantically. Any other VI with the same features and insertion context, i.e., $\varnothing$, could equally be inserted here:

$$
\begin{aligned}
& \text { a. }\left\{\left\{\text { taugh } / \mathrm{T}_{[\mathrm{PST}]}[\mathrm{]}] \text { teach }\right\}, \Sigma_{\mathrm{TEACH}}\right\} \leftrightarrow \varnothing \\
& \text { b. }\left\{\{\text { look }\}, \Sigma_{\mathrm{LOOK}}\right\} \leftrightarrow \varnothing \\
& \text { c. }\left\{\{\text { cat }\}, \Sigma_{\mathrm{CAT}}\right\} \leftrightarrow \varnothing
\end{aligned}
$$

By hypothesis, there is no other minimally specified VI fitting this context, i.e., no VI mentioning $v$, let alone mentioning also $\mathrm{T}_{[\mathrm{PST}]}$. 
Upon selection of the VI in (18), each of the interfaces inspects one of the two sets, $\Phi$ and $\Sigma$, for Exponent and Seme Insertion, respectively. In the case at hand, the allomorph caugh is inserted, via the Elsewhere Principle, over the default allomorph catch. This is because caugh is more highly specified than catch: it refers to a context that matches the context at hand: $\mathrm{T}_{[\mathrm{PST}]}[\mathrm{C}$. Importantly, the allomorph may refer to $\mathrm{T}_{[\mathrm{PST}]}$ as an adjacent terminal node triggering its insertion, since the syntactic structure actually provides adjacency between that node and the point of insertion $(v)$. This is predicted by the RiF theory, in which roots are not nodes in the syntactic configuration, but VIs inserted into nodes. In this case, the node for insertion is directly adjacent to the trigger. The theory allows the catch/caugh difference to be analysed as run-of-the-mill allomorphy triggered in a strictly local and cyclic fashion.

By contrast, assuming the scenario in (13)b, in which the root is a syntactic node, the categorizer intervenes between it and the functional trigger, F. F is thus not adjacent to the insertion node, i.e., the root node. This theory must thus relax the conditions for allomorphy to be triggered, by positing a VI for caugh that makes reference to a nonadjacent node. This is the take in Merchant (2015), based on patterns of rootallomorphy in Modern Greek that allegedly violate the adjacency condition. ${ }^{8}$

On the other hand, in theories like Embick (2010), which adopt the scenario in (13)b and adhere both to an adjacency condition on allomorphy and to the assumption that Vocabulary Insertion takes place bottom-up and node by node, the morphological variation of roots as triggered by morphosyntactic features cannot be handled through Vocabulary Insertion and in a cyclic fashion. Note that this kind of theory sees the root in caught as phonologically adjacent to the tense node, by virtue of the nullness

of the categorizer $($ caugh- $\varnothing-t)$. However, given the cyclicity assumption, when Vocabulary Insertion takes place at the root node the phonological information of $v$ has not been retrieved yet, and so $v$ should block any allomorphic interaction between the root and $\mathrm{T}$, independently of its final phonological realization.

An alternative way to handle root variation like catch/caugh is the post-morphological mechanism of readjustment rules, developed in Embick \& Halle (2005) or Embick (2010), among other works. One of the main empirical claims in Embick \& Halle (2005) is that variation in morphological form is of two distinct types: suppletive variation, determined at Vocabulary Insertion, and variation due to readjustment of a basic phonological representation. Roots are argued not to be subject to Vocabulary Insertion and hence are predicted to only show variation of the latter type. This is claimed to be an empirical fact, and clear cases of suppletion like go/wen(- $t$ ) are argued to involve not roots, but functional material - a light verb, in this case. The formal variation that roots frequently show, like catch/caugh(-t), sing/sang/sung, tooth/teeth, etc., is accounted for via readjustment rules: "phonological rules" invoked

\footnotetext{
${ }^{8}$ See Christopoulos and Petrosino (2018) for a critique of Merchant's (2015) span-based approach to allomorphy in verbal inflection in Modern Greek, and the proposal of an alternative one in terms of Fusion. One of the main reasons for which these authors criticize Merchant's analysis is its failure to predict that overt categorizers like MGr. -iz '-ize' block root allomorphy triggered by functional material (see Marantz 2013). We hasten to point out that this fact is predicted by our theory, trivially. Thus, in verbs with overt categorizers, like crystallize, two roots are involved, CRYSTAL and IZE, the latter intervening between the former and any functional node added. The functional node is therefore expected not to be able to trigger allomorphy in the root.
} 
to modify the form of roots in particular morphosyntactic environments. For instance, after the cycle of Vocabulary Insertion has been run, one such rule would replace the /æt $\int /$ portion of catch with /o:/ in the context of past tense.

Readjustment rules face several empirical and theoretical problems. Empirically, the claim that roots do not show suppletion has been cast doubt upon. For instance, Bobaljik \& Harley (2017) provide evidence that a sizeable group of verbs in Hiaki are truly suppletive, their variation being governed by the number features of the internal argument. We show some of these roots in (20) (from Bobaljik \& Harley 2017:145, ex. 10): (20)a shows intransitive verbs, and (20)b, transitive verbs. The first forms are those adopted by the verb when appearing with a singular internal argument, while the second forms show up with plural internal arguments - subjects in the case of (20)a and direct objects in the case of (20)b:

(20) a. weeye/kaate 'go, walk', weama/rehte 'walk around', kikte/hapte 'stand up', muuke/koko 'die

b. kecha/ha'abwa 'stand (something) up', yecha/hoa 'put down, place', kivacha/kiima 'bring in', me'a/sua 'kill'

In any case, the group of verbs contains specimens, like 'walk around', hardly qualifying as light verbs. ${ }^{9}$

On the theoretical side, readjustment rules are substantially different from usual phonological rules. First, they are restricted to apply to sets of lexical items, often singletons: eat/ate, choose/chose, child/childr-en, etc. Thus, even when they come closest to a phonological rule - as with fricative voicing in plurals of the wolves type - they involve lexical idiosyncrasy, which might as well be handled through the mechanism that was devised, after all, to treat lexical idiosyncrasy: the Vocabulary. Moreover, as pointed out by Bermúdez Otero (2012), unlike usual phonological rules, readjustment rules are sensitive to morphosyntactic information, which must therefore be encoded as part of the rule: for instance, the rule changing $/ \mathrm{I} /$ to $/ \mathfrak{x} /$ in roots such as sing in the context of past tense. ${ }^{10}$

The RiF approach allows us to dispense with readjustment rules and to treat suppletive and non-suppletive root allomorphy as any other kind of allomorphy: through the mechanism of Exponent Insertion, and in a strictly cyclic fashion.

\subsection{Locally determined root allosemy}

Under the default assumption that allosemy is conditioned by the same restrictions as allomorphy (Marantz 2013), the representations in (13) also make different predictions with respect to semantic interpretation. Under (13)a, F may activate a particular alloseme of the root, since there is no intervening node. There are certain phenomena that seem to confirm that the first inflectional projection above the categorizing head may trigger root allosemy. Consider for instance the following nouns in Spanish:

\footnotetext{
${ }^{9}$ See Veselinova (2016) for an in-depth survey of suppletion in verbs.

${ }^{10}$ See more critical remarks in Bermúdez Otero (2012), Siddiqi \& Haugen (2013), and Merchant (2015).
} 


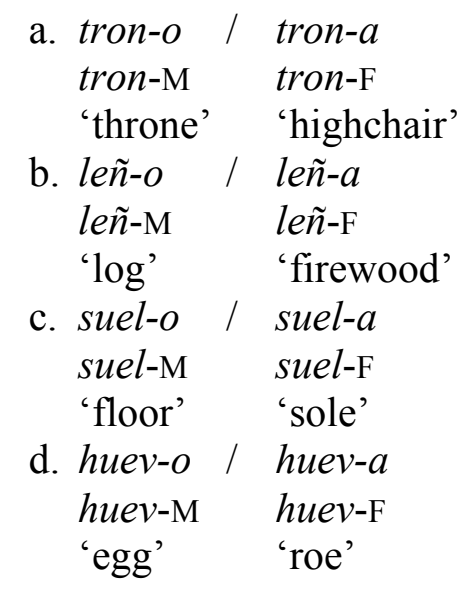

These pairs of nouns share the root, but are distinguished by gender, as marked through the $-o /-a$ alternation. ${ }^{11}$ Importantly, their meaning is different, and that difference, while evidently associated to the change in gender, is unrelated to biological sex, as is the case in other pairs like lob-o/lob-a 'male/female wolf'. Moreover, no generality can be drawn as to the meaning of the masculine counterparts and the feminine counterparts, that is, gender itself is not interpreted. Last, the meanings of the members of each pair are however close enough to each other so as to preclude homonymy, i.e., the use of actually different roots: e.g., TRON 1 'throne' vs TRON 2 'highchair'. The conclusion is that the root is interpreted differently according to whether the gender is masculine or feminine. This fact can be modeled as root allosemy contextually determined by the choice of gender, an inflectional category.

Unlike Acquaviva (2009), we consider gender an independent projection of the noun, and not just a feature of the $n$ categorizer. Evidence for the morphological distinction between gender and $n$ comes from overt categorizers like -ismo or -ura (see Section 3 ). These categorizers overtly show that a particular portion, -ism/-ur, corresponds to the nominalizer, i.e., $n$, while $-o /-a$ corresponds to gender. Furthermore, in ironic speech even an evaluative suffix may appear between both, as in comun-ism-it-o 'little communism' or gord-ur-it-a 'little fatness'. Following Picallo (2008), we take gender to constitute a functional projection directly above the noun, and hosting the feature $[ \pm$ FEM]:

$$
\left[\mathrm{GenP} \operatorname{Gen}_{[ \pm \mathrm{FEM}]}[\mathrm{nP} \mathrm{n}]\right]
$$

Let us take now a closer look at how the allosemic effects of (21) emerge. In the derivation of trono 'throne' and trona 'highchair', the root TRON is inserted in the n, node. Its VI, shown below, specifies the two allosemes "highchair" and "throne":

$$
\left\{\{\text { tron }\},\left\{\text { “highchair”/ Gen }{ }_{[+\mathrm{FEM}]}\left[\_\right], \text {"throne” } \operatorname{Gen}_{[-\mathrm{FEM}]}\left[\_\right]\right\}\right\} \leftrightarrow \varnothing
$$

\footnotetext{
${ }^{11}$ While we acknowledge, with Harris (1991) that the relationship between gender and "word marker" or declension class in Spanish is evidently not one to one, we do not find morphological evidence that these two categories correspond to two different positions, i.e., morphemes. As such, the relationship between them can be thought of as one involving the morpheme Gender, endowed with the morphosyntactic feature $[\mathrm{FEEM}]$, and its exponent, i.e., the word marker. This in turn can be the default one ( $a$ for [+FEM], $o$ elsewhere), or it might be determined by the root through allomorphy (cf. Gen[+FEM] realized as $u$ with root TRIB: tribu 'tribe').
} 
At Vocabulary Selection this VI can be selected for insertion at $n$. The semantic component inspects the set $\Sigma_{\text {TRON }}$ and retrieves the alloseme "highchair" whenever the feature in Gen is [+FEM]. Crucially, the selection of "highchair" is possible precisely because no node is expected to intervene between the root and Gen, as entailed by the $\mathrm{RiF}$ approach. The seme for "highchair" may therefore refer to $\mathrm{Gen}_{[+\mathrm{FEM}]}$ for its retrieval. $^{12}$

Again, theories supporting scenario (13)b cannot derive these effects in a completely local fashion, since the trigger of allosemy (Gen) is not adjacent to the root. Moreover, proposals like that put forward by Marantz (2013) present an additional problem. Marantz (2013) proposes that just as null morphs do not count as interveners for allomorphic interactions at the morphological component (cf. $v$ in caugh- $\varnothing-t$ ), we expect semantically vacuous morphemes to behave alike with respect to allosemic interactions. Among other examples, he refers to cases like pulverized lime. In the participle pulverized, the categorizer $v$, realized as -ize, is semantically vacuous, since no event is necessarily entailed: "pulverized lime can mean "lime in powder form" without the implication of crushing" (Marantz 2013: 111). Marantz suggests that the participial head, -ed is able to trigger an alloseme of the root across the semantically inert categorizer $v$. The whole is merged with a null $a$ head, responsible for the adjectival category:

\section{[ap a [PartP Part [vp [v v PULVER]]]]}

We note that the same explanation cannot be extended to the cases in (21). In these cases, $n$ is not semantically vacuous, but receives an interpretation: "entity". From Marantz's (2013) perspective it is difficult to account for the allosemic effects of (21), triggered by the Gender head in the root across a semantically active categorial head. In our approach no such problem emerges, simply because there is no intervener between Gender and the $\operatorname{root} / n .^{13}$

\footnotetext{
${ }^{12}$ The attentive reader should have noticed that there is nothing in the system precluding the insertion of just any root into a [+FEM] or a [-FEM] environment. Nouns that show up with either gender, like lob-o 'male wolf' / lob- $a$ 'female wolf' or the ones in (20), are naturally accommodated in this system. However, this architecture also generates abnormal items like masculine \#el meso (instead of feminine la mesa 'the table') or feminine \#la techa (instead of masculine el techo 'the ceiling'). We in fact believe that these expressions should be generated by the grammar, since they are found in jocular language: cf. el coso (M), instead of la cosa (F) 'the thing', el palabro (M), instead of la palabra (F) 'the word', la marida (F), instead of el marido (M) 'the husband', etc., and productively in the expression iNi ROOT(M/F) $n i$ ROOT(F/M)!, lit., "neither ROOT(M/F) nor ROOT(F/M)" 'Don't talk to me about the X!': iNi mesa ni meso! 'Don't talk to me about the table!', iNi techo ni techa! 'Don't talk to me about the ceiling!' (thanks to Antonio Fábregas and Mercedes Tubino for some of these examples). The awkwardness of meso and techa is due to the fact that their VIs are not endowed with any seme allowing their interpretation in a masculine and a feminine gender context, respectively. Thus, we believe that the oddness of these expressions cannot be put on a par with cases of violation of grammatical gender agreement as *la mesa es blanco 'the table(F) is white(M)'. More generally, we are advocating for the idea that nouns in Romance (and other languages) are only interpretable in the context of grammatical gender.

${ }^{13}$ An anonymous reviewer is right in wondering how the "entity", LF-related meaning of $n$ coexists with the conceptual meaning of the root inserted therein. The same question should of course be asked about the rest of the categorizers. First, we note that we concur with Acquaviva $(2008,2009)$ that roots are only interpretable in the presence of functional structure, and, in this sense, we identify ourselves with theories like his, Borer's (2005), or AUTHOR1 \& ANOTHER AUTHOR (2014), in not positing
} 
A further prediction made by our system is that functional projections above Gender, and in particular, Number, should not constitute possible contexts for the interpretation of the noun. As far as we know, this seems to be true for Spanish and Catalan. In previous versions of this research, we actually considered cases like the one below as evidence that Number - plural, in this case- could trigger the retrieval of a particular alloseme of the root:
a. El padre de Inés es Juan/\#Sole. the padre of Inés is Juan(M)/\#Sole(F) 'Inés's father is Juan/\#Sole.'
b. Los padre-s de Inés son Juan y Sole. the padre-PL of Inés are Juan(M) and Sole(F) 'Inés's parents are Juan and Sole.'

The word padre in the singular can only mean 'father' ((25)a), while it may mean 'parent' in the plural $((25) b)$. At first sight the plural morpheme could be taken to trigger an alloseme 'parent' in the root PADR. However, the special interpretation of padre in (25)b cannot be an allosemic effect. First, the sense 'parent' is optional in padres, which may also mean 'fathers', while the interpretations induced by gender in (21) are unavoidable. More importantly, the sex-neutral interpretation of the masculine in a plural context is possible with other roots referring to masculine kinship terms, like abuelos 'grandfathers'/'grandparents', tios 'uncles'/'uncle and aunt', etc. Even more generally, masculine plurals referring to animate beings can denote either a group of males or a group of males and females: profesor-es 'male teachers'/'teachers', norueg-o-S 'male Norwegians'/'Norwegians', etc. The optionality and generality of this semantic effect suggests that it cannot be accounted for by any resort to idiosyncratic properties of particular roots (i.e., stored semes, in our theory), but to some other conceptual operation. ${ }^{14}$

\section{Exploring semi-lexicality}

In this section we briefly explore a possibility predicted by our approach: the insertion of roots in functional heads distinct from categorizers. We do so through the analysis of numeral classifiers. In section 5.1, we claim that classifiers correspond to roots that give exponence and conceptual content to the set of functional heads involved in

semantic types for roots - unlike Harley (2005), Marantz (2013), or Levinson (2014). Our system derives the non-interpretability of roots in the absence of functional structure naturally, simply because it cannot yield bare roots as output for LF (or PF). We think that LF automatically assigns an "entity" meaning to $n$, while the root can only be interpreted as associated to this LF meaning. For instance, [nP $\mathrm{n} \diamond \mathrm{DOG}]$ is interpreted, informally, as "dog-entity", while [ $\left.{ }_{\mathrm{vP}} \mathrm{V} \diamond \mathrm{DOG}\right]$ is interpreted as "dog-event". True, since the relationship between the categorizer and the root is not syntactic, we cannot invoke any known semantic rule - functional application? - to generate these interpretations. Rather, we think that the conceptual content of the root is identified with the interpretation of the categorizer: "an entity/event that is dog".

${ }^{14}$ In a linguistic system in which Number were structurally adjacent to $n /$ ROOT, nothing would prevent it from triggering special allosemic interpretations. The semantically idiosyncratic plurals in Italian studied by Acquaviva [2008:123ff., 2009], like membr-o(M.SG) 'member, of a committee, for instance' / membr-a(F.PL) 'limbs' could constitute a case in point. Since, as pointed out by Acquaviva, Gender and Number are morphologically undistinguishable in this language, we expect one and the same morpheme to be adjacent to the $n$ /ROOT node — perhaps following a Fusion operation, as proposed by this author - and thus to be able to trigger a special interpretation thereof. 
counting ad individuation in languages where these inflectional heads do not count with a dedicated VI. ${ }^{15}$ This hypothesis allows us to derive a classical observation found in the literature and explored in section 5.2. That is, that the availability of classifiers correlates with the lack of inflectional number morphology (cf. Greenberg [1972]; also Li [1999] for Chinese and Löbel [2001] for Vietnamese). Finally, we provide an analysis of classifiers as roots into a functional node.

\subsection{Classifiers involve roots, not nouns.}

In this section, we provide evidence to support the claim that classifiers are roots into functional nodes. First, we show that classifiers serve a syntactic purpose, but also contain encyclopaedic information. Second, classifiers conform a (semi-)open class. We show these two properties in turn by mainly providing examples from Mandarin Chinese. Finally, we provide arguments to support the analysis of classifiers as roots and not nouns.

Aikhenvald [2000] shows that the relation between classifiers and the nouns that they classify is culture-specific in most languages. The selection of classifiers depends on properties associated with the speakers' world knowledge. For example, in Mandarin Chinese the noun huanggua 'cucumber' can select either gen, the classifier for multidimensional long and rigid objects, or tiao, the classifier for unidimensional long objects. Speakers seem thus to select the classifier depending on the most salient properties of the object denoted by the classified noun.

That classifiers are associated with an encyclopedia entry, that is, a non-empty set $\Sigma$, is supported by the existence of polysemy in classifier systems. For example, classifier zhi carries three types of lexical meanings depending on the type of noun that it classifies: it can function as a generic classifier for animals, as a classifier for elements that come in pairs and as classifier for things that have volume (Zhang [2007]). This case of polysemy receives a straightforward explanation if we assume that $z h i$ has an encyclopaedic entry, as in (26).

(26) zhi: $\{\Phi, \Sigma\}$, where $\Sigma=\left\{\sigma_{1}, \sigma_{2}, \sigma_{3}\right\}$, in which $\sigma_{1}=\{$ animal $\}, \sigma_{2}=\{$ one in a pair $\}$, $\sigma_{3}=\{$ ball shape $\}$.

Classifiers illustrate clearly the phenomenon of semi-lexicality (Corver and Van Riemsdijk [2001]) because they encode two types of meaning. First, as shown in the vast literature of number classifiers, they allow the individuation in the interpretation of nouns that allow counting or other referential mechanisms. But also, count classifiers do more than that: they contain additional, lexical meaning, that is interpreted as serving a qualifying function to the interpretation of the noun (see Zhang [2007]). The semilexicality of these elements can be seen in the fact that although count classifiers appear obligatorily in numeral expressions, the choice of classifier can vary according to semantic purposes. That is, contrary to what happens with inflection, classifiers can be used for creating new meanings with stylistic purposes. See the examples below from Zhang [2007: 54]: the object that the NP refers to is always the same, but the choice of classifier serves to encode semantic qualities such as formality, positive or negative

\footnotetext{
${ }^{15}$ See Acquaviva (2009) for a similar view on classifiers and semi-lexical item. However, Acquaviva argues that the root provides only a phonological exponent to the semi-lexical item, and no encyclopedic content, contrary to the evidence reviewed in this section.
} 
feelings towards the object etc. The stylistic difference in the use of the classifier depends on the specificity of the classifiers. Thus, zhang is a general classifier for all types of flat objects, $f u$ is a specialised classifier for paintings and clothes, and zhen can only be used with paintings.
a. yi zhang hua
One CL painting
'a painting [with a flat surface]'
b. $y i \quad f u \quad h u a$
One CL painting
'a painting [indicates expertise]'
$\begin{array}{lll}\text { c. } y i & \text { zhen } & \text { hua } \\ \text { One } & \mathrm{CL} & \text { painting }\end{array}$
'a painting [indicates that the painting is valuable]'

Zhang [2007] also shows that speakers do not always agree regarding the choice of classifiers. In this respect, Mandarin Chinese contrasts with languages with inflectional number systems, which are rigid. That is, in Spanish, speakers that intend to denote plurality cannot choose the type of morpheme they insert. In contrast, in Chinese, speakers are required to use a classifier if they are counting an object, but they have a choice with respect to which one to use.
a. dos libro-s $/{ }^{*} \varnothing$
two book-PL-SG
b. tres $\operatorname{ros} a-s / *_{-} \varnothing$
three rose-PL/SG

A prediction that our model does regarding semi-lexical elements is the existence of information regarding the syntactic construal of the functional projection and semantic information included in an Enclyclopedic entry.

Classifiers can be shown to conform a (semi-)open class category. The inventory of classifiers in classifier systems is limited, but wide, the majority of classifier languages containing from 15 to more than 100 classifiers. ${ }^{16}$ The number of classifiers in Chinese according to Zhang [2007] reaches 902.

That classifiers form open systems is also made evident in other classifier systems, by the phenomenon of repeaters. Tai, for instance, show this type of classifier system in which when a new noun is adopted, the same noun acts as its own classifier (example from Kolver [1983: 190], apud Aikhenvald [2000]):

$$
\text { pratheet saam pratheet }
$$

\footnotetext{
${ }^{16}$ There are languages with quite restricted systems of classifiers. For example, in Khasi there are only two classifiers, as shown in (i): for animate and inanimate entities (Joseph [2009]). We think that systems of this kind involve inflectional (or derivational) gender or number, rather than classifiers:
(i) a. ar-ngut $\mathrm{ki}$ briew
two-person ART man
'two men'
b. ar-tylly $\mathrm{ki}$ maw
two-thing ART stone
'two stones' 
land three CL(land)

'three countries'

It seems to us that the limitations on the inventories of classifiers must be due to nongrammatical reasons. We explore this in section 5.3 where we define the semantic interpretation of roots into functional nodes.

Although it is clear that classifiers involve a root, we have not proved yet that they are not just nouns modifying another noun. To test this hypothesis, we need to see if classifiers accept adjectival modification, as nominals do. According to Cheng and Sybesma [1999], Borer [2005], and Cowper and Currie Hall [2012], unlike nouns, classifiers do not accept adjectival modification, with some exceptions.

$$
\begin{array}{cccl}
\text { a. *san } & \text { da } & \text { ge } & \text { ren } \\
\text { three big } & \text { CL } & \text { people } \\
\text { b. *yi da } & \text { zhi } & \text { gou } \\
\text { one big } & \text { CL } & \text { dog } \\
\text { c. *yi da } & \text { wei } & \text { laoshi } \\
& \text { one big } & \text { CL } & \text { teacher }
\end{array}
$$

As pointed out before, there are some exceptions to the claim that classifiers do not accept adjectival modification. A closer examination though reveals that these exceptions have a different interpretation and structure. Thus, Cheng and Sybesma [1999] shows that classifiers that allow adjectival modification should be called massifiers, to distinguish them from count classifiers. Massifiers behave very much like measure phrases in languages that do not have classifiers, as in the example (31) from English. That is, they can combine with either mass and count nouns to denote a quantity or a container, respectively. For example, a noun like shu 'book' can combine with either a count classifier to denote an object (32)b, or a massifier to denote the container in which a set of books is located, (32)a.
(31) a. A book
b. A box of books.
(32) a. Yi xiang shu
One CL book
'One box of books'
b. $Y i$ ben shu
One CL book
'One book'

That massifiers are more akin to nominal structures such as the ones we find in non-classifiers languages can again be seen by the fact that nominal quantifiers such as Catalan un tou 'a lot of' or un grapat 'a bunch' also allow adjectival modifiers, with similar types of adjectives and similar interpretation.

$$
\begin{aligned}
& \text { a. } y i \quad d a \text { zhang zhi } \\
& \text { one big CL-sheet paper } \\
& \text { 'one large sheet of paper' }
\end{aligned}
$$

b. na yi xiao xiang shu 
that one small CL-box book

'that one small box of books'

c. Un bon grapat / tou de cebes.

A well bunch mass of onions

'A lot of onions'

d. un petit grapat de llibres $i$ papers antic

a small bunch of books and papers old

'a small pile of books and old papers'

To conclude, we have presented evidence that count classifiers of the type found in Mandarin Chinese contain a root because they conform (semi-)open class systems, and convey encyclopaedic information besides serving a functional purpose. In addition, we have provided evidence against the analysis of classifiers as nominals, and in favor of the view that they are roots into functional nodes. ${ }^{17}$

\subsection{Classifiers correlate with the lack of inflectional number morphology.}

As discussed in section 2, the RiF approach conceives roots as VIs that are ontologically similar to inflectional and derivational morphemes. One of the predictions that we make is that in languages in which there is no morpheme to give exponence to a particular functional node, a root can be inserted. Thus, we claim that this is precisely what happens in languages that have number classifiers. If our hypothesis is on the right track, we should not expect to have languages with both inflectional number and classifiers. This prediction seems to be supported by the classical Greenberg-Sanches's generalisation that classifiers and inflectional number morphology are in complementary distribution (Greenberg [1972], and after him, Li [1999], Löbel [2001], Cheng and Sybesma [1999], Borer [2005], and Cowper and Currie-Hall [2012], among many others), and that we label as the Number-Classifier complementarity. As argued in Borer [2005] and Cowper and Currie-Hall [2012] the

\footnotetext{
${ }^{17}$ There are some counterexamples to the claim that count classifiers do not accept adjectival modification. For example, Li [2011] and Zhang [2012] claim that count classifers do accept adjectival modification in some contexts. However, we believe that these examples are not real counterexamples to the claim sustained here. First, it is not clear that the examples of modified count classifiers are indeed count classifiers and not massifiers, as most classifiers can function as both (Zhang [2007], see also $\mathrm{Li}$ [2011]). Second, it seems clear that in the rest of examples found with count classifiers, the adjective is not modifying the classifier, or the noun. The reading of these adjectives is similar to the function that diminutives or augmentatives have in languages with evaluative derivational morphology (see Li 2011). We leave for now the exact analysis that these constructions should have, but it is clear that they do not behave as regular adjectival nominal configurations, compare (i) and (ii), from Li
} [2011].

(i) a. ji li xiao huanggou. several Cl-grain small soybean 'several grains of small soybean'

b. $j i$ li hen xiao de huangou several Cl-grain very small Mod soybean 'several grains of very small soybeans'

(ii) a. mei-ge xuesheng dou jiang le yi ge da beizi. every- $\mathrm{Cl}$ student all award Perf one $\mathrm{Cl}$ big mug

b. mei-ge xueshengdoujiang le yi da gebeizi. every- $\mathrm{Cl}$ student all award Perf one big $\mathrm{Clmug}$ 'Every student is awarded with a big mug.' 
complementarity appears to be both distributional and functional: both number and classifiers are involved in the mechanisms that allow counting. In this subsection, we explore the validity of this claim.

Greenberg [1972] attributes to Sanches [1971] the following generalisation: "classifier languages do not have compulsory expression of nominal plurality, but at most facultative expression (Greenberg [1972:17])". Greenberg extends this observation by Sanches to the correlation that classifier languages lack number marking. This generalisation has been supported by the observation that classifiers and number marking do not concur in most languages of the world. A recent quantitative study that uses WALS database has shown that there are few exceptions to this complementarity (Her and Chen [2013]). Our approach predicts exactly this picture.

Some authors sustain a weaker version of the Number-Classifier complementarity to include the few counterexamples found. Borer [2005], and Cowper and Currie-Hall [2012], among many others claim that number marking and classifiers can coexist in one language, provided they appear in complementary distribution, since they express the same type of features. In our approach, the existence of such languages is unexpected since we predict that a root will never be inserted in contexts for which there is an inflectional morpheme. In this section, we review evidence that classifiers are indeed not compatible with inflectional number systems, but they can coexist with non-inflectional number markers, that involve other types of morphological or syntactic processes. That is, adopting Wiltschko's $(2008,2009)$ view of noninflectional number, we will interpret the non-contrastiveness of plural markers as evidence that the number system is not inflectional. In doing so, the GreenbergSanches generalisation holds without exception, which we reinterpret as a complementarity between classifiers and inflectional number.

A typical counterexample to the strong Number-Classifier complementarity is Armenian (Borer [2005], Bale and Coon [2014]), which I copy below from Borer [2005:95]. Armenian has both classifiers and number markers, although they are never found in the same NP (34)b. ${ }^{18}$

$\begin{array}{lll}\text { a. Yergu had hovanoc } & \text { uni-m } \\ \text { Two CL umbrella } & \text { have-1SG } \\ \text { 'I have two umbrellas' } & \\ \text { b. Yergu hovanoc-ner } & \text { uni-m } \\ \text { Two umbrella-PL } & \text { have-1SG } \\ \text { 'I have two umbrellas' } & \\ \text { c. *Yergu had hovanoc-ner } & \text { uni-m } \\ \text { Two CL umbrella-PL } & \text { have-1SG } \\ \text { 'I have two umbrellas' } & \end{array}$

\footnotetext{
${ }^{18}$ Borer [2005] analyses this case as an illustration that plural and classifiers are two different types of exponents for the same functional node. Although we agree with this approach accounts for crosslinguistic complementarity, we remain agnostic for intralinguistic complementarity. See Bale and Khanjian [2009] for compelling arguments in favor of a semantic approach to explain the distribution of classifiers and plural in Armenian.
} 
However, if we follow Wiltschko's (2009) observation that plural marking is not contrastive in languages without inflectional number, Armenian is not a real counterexample. As in the Halkolemem Salish case, Armenian plural marker is not contrastive, as shown in (35) from Bale and Khanjian [2009, 2014].

$$
\begin{aligned}
& \text { a. Yergu dUgha vaze-ts. } \\
& \text { Two boy(SG) run-PST } \\
& \text { 'Two boys ran.' } \\
& \text { b. Yergu dUgha-ner } \\
& \text { two vaze-ts-in. } \\
& \text { 'Two boy-PL ran.' }
\end{aligned}
$$

The absence of the plural marker -ner does not trigger a singular interpretation but a neutral interpretation regarding number. There are other alleged counterexamples, very similar to Armenian. As shown in Cowper and Currie Hall [2012] for Persian and Korean, Kwon and Zribi-Hertz [2004] for Korean, plural markers in those languages plural markers are not contrastive, contrary to inflectional number markers. More counterexamples are discussed in Her and Chen [2013], with similar findings.

Finally, we briefly comment one more counterexample that does not fit in the previous picture, and that involves Mandarin Chinese: the existence of a pluraliser suffix -men. Again, we argue, following Li (1998) that this suffix is not a true inflectional marker of number. ${ }^{19}$ Mandarin Chinese shows a pluraliser suffix -men that only appears in pronouns and in nouns, and that is postponed to the noun.

$$
\begin{aligned}
& \text { Wo qu zhao haizi-men } \\
& \text { I go find child-men } \\
& \text { 'I will go find the children' }
\end{aligned}
$$

Pluraliser -men only appears with NPs (and pronouns) that refer to animate beings, commonly humans. Moreover, it is not contrastive in nominals. The examples below do not have any pluralizer but are compatible with a plural interpretation of the nominal.

$$
\begin{aligned}
& \text { Wo qu zhao haizi } \\
& \text { I go find child } \\
& \text { 'I will go find some children or the child' }
\end{aligned}
$$

As pointed out in Li [1999] -men means more than plurality in Mandarin Chinese because it has always a strong definite interpretation. Adopting Li's [1999] analysis, men spells out not only a semantics of plurality but also features of Person, which can be argued to be above D (Hinzen and Sheehan [2013]). If this is the case, we can hypothesize that -men is not a Number marker, but an element that spells out person features and that is also associated with a meaning of plurality, but not with an

\footnotetext{
${ }^{19}$ Cantonese has also an alleged pluraliser marker $d i$ that has been argued to be a counterexample to the Number-Classifier complementarity. See Cowper and Hall [2012] for arguments in favor of an analysis $d i$ as a massifier with an indefinite interpretation.
} 
inflectional suffix. ${ }^{20}$

In this section, we have seen that the Number-Classifier complementarity defined as a complementary distribution between inflectional plural and classifiers is solid typologically. The few counterexamples found in the literature are not cases of inflectional number systems, as shown for Armenian, Persian or Korean. In the two cases discussed in the literature concerning Mandarin and Cantonese Chinese there are strong arguments that supports the analysis that they are quantifiers or determiners associated with a meaning of plurality, but not plural inflectional morphemes. To conclude, the $\mathrm{RiF}$ approach makes the correct typological prediction regarding number and classifier systems across languages. In next section, we propose an analysis of classifiers following this approach.

\subsection{Classifiers in the Root-into-functional node approach}

In this section we develop an analysis of classifiers as roots that give both exponence and conceptual content to a functional node, DivP in our view. In doing so, we provide an explanation for the phenomenon of semi-lexicality, that is, the phenomenon by which a functional word may serve both to provide syntactic construal and encyclopaedic conceptual information.

First, we need to explain our assumptions on the syntactic structure of NPs, specifically regarding number and individuation. We assume Borer [2005] approach to the syntax and semantics of NPs, by which inflectional plural and classifier systems are two morphological manifestations of a uniform syntactic structure. With Borer [2005], we assume that all roots have a mass denotation and that nouns are rendered countable by combining with a functional projection that provides portions to their denotation. This functional projection is called DivP. Dominating this projection, there exists NumP, which assigns quantity to portions and is responsible for counting. In a language with inflectional number, the plural marker is a VI that gives exponence to Div, a functional projection that partitions the denotation of the nominal and allows counting by Num. I provide the structure of English singular one cat, plural cats, following Borer (2005).
a. [Nump [one]Num [DivP [one] Div $\diamond \varnothing[\mathrm{nP} \mathrm{n} \hookleftarrow \mathrm{CAT}]]]]$
b. [Nump Num [DivP Div $\diamond S[\mathrm{nP} \mathrm{n} \hookleftarrow \mathrm{CAT}]]]]]$

In classifier languages, no VI is specified for Div. In these cases, our model predicts that a root will be inserted. This is what happens with classifiers in Mandarin Chinese. We provide the derivation for yi ben shu "a book" and yi tiao she "one

\footnotetext{
${ }^{20}$ As in the Armenian case, -men never concurs with classifiers, although this may have nothing to do with the number- classifier complementarity.

(i)

$\begin{array}{lll}* \text { san } & \text { ge } & \text { xuesheng-men } \\ \text { Three } & \mathrm{cl} & \text { student-men }\end{array}$

As argued in Li (1999), the postpositional order of me could be due by movement of $\mathrm{N}$ into the specifier of DP. Classifiers are not possible with -me, because they would intervene in this type of movement. The specific analysis of $m e$ is out of the purposes of this work and deserves an investigation that we cannot undertake here. See Li (1998) for a detailed analysis.
} 
snake' in Chinese below: ${ }^{21}$
a. yi tiao she
one CL snake
'one snake'
b. san ben shu
three CL book
'three books'

$$
\text { [NumP [NP yi/san] [Num' Num [DivP Div } \hookleftarrow \mathrm{TIAO/BEN} \mathrm{[nP} \mathrm{n} \hookleftarrow \mathrm{SHE} / \mathrm{SHU}]]]]
$$

That is, in the examples in (19), classifiers tiao and ben are two roots, namely, two pairings of sets of phonological and semantic representations that are inserted via Vocabulary Insertion by competition. As no VIs are specified with the syntactic features of counting and division in the language, by the Subset Principle, roots end up giving exponence.

The semantic interpretation of roots in Div nodes follows the same mechanism that drives the interpretation of roots into categorizer nodes, see Author 1 \& Author 2 (2014) and footnote 13. That is, the root is interpreted through the functional node Div that assigns a meaning of individuation at LF. Let's see a concrete example: classifier tiáo is interpreted as 'an individuator for things that have a long shape' when used as a count classifier. This interpretation is the result of interpreting the seme that denotes 'long shapeness' through the LF interpretation of Div, that provides the meaning of portioning masses. The type of semantic interpretation will not be syntactic since insertion is post syntactic in our model. Thus, we posit a level of semantic integration in which the semes associated with set $\Sigma$ are recovered and integrated with the syntactic construal provided by the LF. The interpretation of the classifier will be a combination of these two semantic components yielding the semilexical flavour of these elements.

This mechanism allows us also to explain the different semantic interpretations that classifiers have depending if they are count classifiers or massifiers. For example, tiáo can function as both. As a count classifier it denotes the shape of the object it classifies, but as a massifier it denotes the shape of the thing that contains the classified noun. As argued in section 5.1, massifiers have a nominal denotation very similar to the one that we find in Measure Phrases in inflectional number languages like English, namely, a bunch of or a box of. In both cases, the encyclopaedic meaning remains the same: in one case it qualifies the shape of the object it classifies, in the second case it qualifies the shape of the container. The examples below illustrate this contrast between massifiers and count classifiers respectively: (41) and (42).
a. Yi tiao xiangyan
One CL cigarettes
'a carton of cigarettes'
b. Yi tiao mianbao.
one $\mathrm{cl}$ bread
'one loaf of bread'

\footnotetext{
${ }^{21}$ We follow Borer [2005] analysis to account for Chinese classifiers. See Li [1998] and Cheng and Sybesma [1999] for arguments in favor of the view that numerals are in NumP or NumeralP (\#P), that we do not justify here.
} 


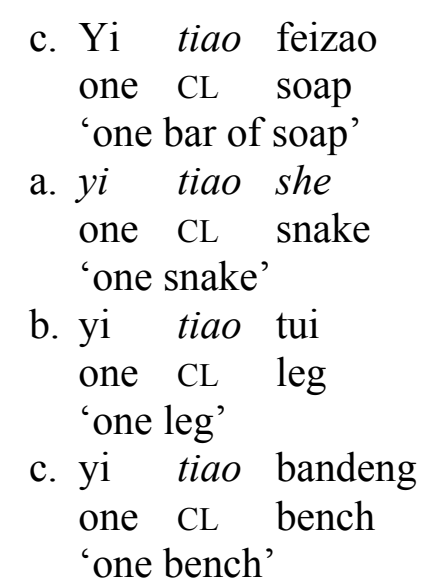

If classifiers are roots spelling out functional nodes, there is still the question of what prevents classifier systems to be true open class systems, not semi-open systems. It seems to us that the limitations on the inventories of classifiers must be due to nongrammatical reasons, such as convention or world knowledge of the speakers. That is, in order for a root to be able to act as an individuator, it must be somehow related with the noun it classifies, by describing a particular physical feature or a particular type of class. Classifiers in Mandarin Chinese fit into these types of meanings: classifiers denote particular sizes, shapes, configurations or establish a taxonomic relation with the nouns that they classify (Allan [977], Tai [1994], Aikhenvald [2000], Lobel [2001], Zhang [2007]). In some cases, the relation between the classifier and the noun is non-transparent as argued in Zhang [2007] and it is more a matter of convention. In general, speakers tend to use more general classifier in non-formal varieties (Zhang [2007]). This trend towards a simplification of the system may not be due to grammatical factors, favoring economy on communications, for example. We hypothesize that there is nothing in the grammar that prevents classifier systems to be open class systems. Indeed this typological option may be what is at stake in languages that have repeaters, like in the examples from Tai above (Kolver 1983: 190, apud Aikhenvald 2000) that we will not be able to explore further in this paper.

\section{Concluding remarks}

In this paper we have explored some predictions of the RiF approach to the integration of roots in the structure, whereby roots correspond to (lately inserted) VIs that phonologically and semantically interpret functional nodes. Roots such as cat or up are VIs that count with no context of insertion and are predicted to be insertable into any node that does not count with a dedicated VI of its own. For instance, they can be inserted into any categorial node $(n, v, a)$. This is why simple words are categorially labile. In some languages roots can also be inserted into inflectional nodes like Number, since there is no more highly specified VI realizing it. We argue that this could be the case of nominal classifiers, which have the hallmarks of roots. On the other hand, derivational affixes like -ation or -al also correspond to roots, in this case roots inserted into little head categorizers, but their VIs have a context of insertion accounting for their categorial rigidity and their c-selection properties. Since roots are directly inserted into functional nodes, this insertion is predicted to be alterable by the functional node merged directly on top. We have provided evidence from allomorphy and allosemy patterns that this is the case. Among other things, the RiF approach allows to eliminate Readjustment Rules. Finally, our approach accounts 
naturally for the phenomenon of semi-lexicality, in which a word expresses both functional and lexical meaning. We have illustrated this account with an analysis of number classifiers, but we believe that it can be extended to other semi-lexical categories in further works.

Our approach makes two interesting predictions on linguistic variation that deserve further exploration. First, it predicts the existence of linguistic types that emerge from the morphological properties of exponents of functional categories like number, gender, tense, aspect and mood. Second, it provides a natural explanation for the existence of grammaticalization processes that govern diachronic change.

\section{References}

Acquaviva, Paolo. 2008. Lexical plurals. A morphosemantic approach. Oxford: Oxford University Press

Acquaviva, Paolo. 2009. Roots and lexicality in Distributed Morphology. York Papers in Linguistics Series 2 (YPL 2) 10: 1-21.

Aikhenvald, Alexandra Y. 2000. Classifiers: A typology of noun categorization devices. Oxford: Oxford University Press.

Allan, Keith. 1977. Classifiers. Language 53. 284-310.

Arad, Maya. 2003. Locality constraints on the interpretation of roots: The case of Hebrew denominal verbs. Natural Language \& Linguistic Theory 21. 737-778.

Bale, Alan, and Jessica Coon. 2014. Classifiers are for numerals, not for nouns: consequences for the mass/count distinction. Linguistic Inquiry, 45(4), 695-707.

Bale, Alan and Hrayr Khanjian. 2009. Classifiers and number marking. In Proceedings of semantics and linguistic theory (SALT) XVIII, p. 73-89.

Bale, Alan, and Hrayr Khanjian. 2014. Syntactic complexity and competition: The singular-plural distinction in Western Armenian. Linguistic Inquiry 45.1 (2014): $1-26$.

Bermúdez-Otero, Ricardo. 2012. The architecture of grammar and the division of labor in exponence. In Jochen Trommer (ed.), The morphology and phonology of exponence, 8-83. Oxford: Oxford University Press.

Bobaljik, Jonathan D. \& Heidi Harley. 2017. Suppletion is local: Evidence from Hiaki. In Heather Newell, Máire Noonan and Lisa Travis (eds.), The Structure of Words at the Interfaces, 141-159. Oxford: Oxford University Press.

Borer, Hagit. 2003. Exo-skeletal vs. endo-skeletal explanations: syntactic projections and the lexicon. In John C. Moore and Maria Polinsky (eds.), The Nature of Explanation in Linguistic Theory, 31-67. Chicago: University of Chicago Press.

Borer, Hagit. 2005. Structuring Sense. Volume I: In Name Only. Oxford: Oxford University Press.

Borer, Hagit. 2013. Structuring Sense. Volume III: Taking Form. Oxford: Oxford University Press.

Bye, Patrik \& Peter Svenonius. 2012. Nonconcatenative morphology as epiphenomenon. In Jochen Trommer (ed.), The morphology and phonology of exponence, 427-495. Oxford: Oxford University Press.

Cheng, Lisa Lai-Shen. 2012. Counting and classifiers. In Diane Massam (ed.), Count and Mass Across Languages, 199-219. Oxford: Oxford University Press.

Cheng, Lisa Lai-Shen, \& Rint Sybesma. 1999. Bare and not-so-bare nouns and the structure of NP. Linguistic inquiry, 30(4), 509-542.

Chierchia, Gennaro. 1998. Reference to kinds across languages. Natural Language Semantics 6: 339-405. 
Christopoulos, Christos \& Roberto Petrosino. 2018. Greek Root-Allomorphy without Spans. Proceedings of the 35th West Coast Conference on Formal Linguistics (WCCFL 35). 151-160.

Corver, Norbert \& Henk C. van Riemsdijk (eds.). 2001. Semi-Lexical Categories. The content of function words and the function of content words. Berlin: Mouton de Gruyter.

Creemers, Ava, Jan Don \& Paula Fenger. 2017. Some affixes are roots, others are heads. Natural Language \& Linguistic Theory 36(1). 45-84.

Cowper, Elizabeth \& Daniel Currie Hall. 2012. Aspects of individuation. In Diane Massam (ed.), Count and Mass Across Languages, 199-219. Oxford: Oxford University Press.

De Belder, Marijke. 2011. Roots and affixes: Eliminating lexical categories from syntax. Utrecht University/UiL-OTS \& HUBrussel/CRISSP dissertation.

De Belder, Marijke \& Jeroen van Craenenbroeck. 2015. How to merge a root. Linguistic Inquiry 46(4). 625-655.

Embick, David \& Alec Marantz. 2008. Architecture and blocking. Linguistic Inquiry (39). 1-53.

Embick, David. 2010. Localism versus globalism in morphology and phonology. Cambridge, MA: MIT Press.

Guimarães, Maximiliano. 2000. In defense of vacuous projections in Bare Phrase Structure. University of Maryland Working Papers in Linguistics 9. 90-115.

Greenberg, Joseph H. 1972. Numeral Classifiers and Substantival Number: Problems in the Genesis of a Linguistic Type. Working Papers on Language Universals 9. $1-39$.

Grimshaw, Jane. 1990. Argument Structure. Cambridge, MA: MIT Press.

Halle, Morris. 1997. Distributed Morphology: Impoverishment and Fission. MIT Working Papers in Linguistics (MITWPL) 30: Papers at the Interface. 425-449.

Harley, Heidi. 2005. How do verbs get their names? Denominal verbs, manner incorporation, and the ontology of verb roots in English. In Nomi Erteschik-Shir and Tova Rapoport (eds.), The syntax of aspect: Deriving Thematic and Aspectual Interpretation. 42-64. Oxford: Oxford University Press.

Harley, Heidi. 2014. On the identity of roots. Theoretical Linguistics 40. 225-276.

Harris, James W. 1991. The Exponence of Gender in Spanish. Linguistic Inquiry 22(1). 27-62.

Harris, James W. 1999. Nasal Depalatalization no, Morphological Wellformedness si; the Structure of Spanish Word Classes. MIT Working Papers in Linguistics (MITWPL) 33: Papers on Syntax and Morphology, Cycle One. 47-82.

Haugen, Jason D. \& Daniel Siddiqi. 2013. Roots and the derivation. Linguistic Inquiry 44. 493-517

Hinzen, Wolfram \& Michelle Sheehan. 2013. The philosophy of universal grammar. Oxford: Oxford University Press.

Joseph, U. V. 2009. The Numeral 'One' in Khasi and Karbi. North East Indian Linguistics 2. 149-210.

Kayne, Richard. 2009. Antisymmetry and the Lexicon. In Jeroen van Craenenbroeck (ed.), The Linguistic Variation Yearbook 2008, 1-32. Amsterdam: John Benjamins Publishing.

Krifka, Manfred. 1995. Common nouns: A contrastive analysis of Chinese and English. In Gregory Carlson and Francis Pelletier, The generic book, 398-411. Chicago: University of Chicago Press.

Kolver, Ulrike. 1982. Klassifikatorkonstruktionen in Thai, Vietnamesisch und 
Chinesisch. In Hansjakob Seiler, Christian Lehmann, Franz J. Stachowiak (eds.), Apprehension: Das sprachliche Erfassen von Gegenständen. Teil I: Bereich und Ordnung der Phänomene, 160-245. Tübingen: Narr.

Levinson, Lisa. 2014. The ontology of roots and verbs. In Artemis Alexiadou, Hagit Borer and Florian Schäfer (eds.), The syntax of roots and the roots of syntax, 208-229. Oxford: Oxford University Press.

Li, Yen-hui Audrey. 1998. Argument determiner phrases and number phrases. Linguistic Inquiry 29 (4) , p. 693-702.

Li, Yen-hui Audrey. 1999. Plurality in a classifier language. Journal of East Asian Linguistics 8 (1). 75-99.

Löbel, Elisabeth. 2001. Classifiers and semi-lexicality: Functional and semantic selection. In Norbert Corver \& Henk C. van Riemsdijk (eds.), Semi-lexical categories, 223-272. Berlin \& New York: Mouton de Gruyter.

Longobardi, Giuseppe. 1994. Reference and proper names: A theory of N-movement in syntax and logical form. Linguistic Inquiry, 609-665.

Longobardi, G. 2001. How comparative is semantics? a unified parametric theory of bare nouns and proper names. Natural Language Semantics, 9(4), 335-369.

Lowenstamm, Jean. 2014. Derivational affixes as roots: Phasal spell-out meets English stress shift. In Artemis Alexiadou, Hagit Borer \& Florian Schäfer (eds.). The syntax of roots and the roots of syntax, 230-259. Oxford: Oxford University Press.

Marantz, Alec. 1997. No escape from syntax: don't try morphological analysis in the privacy of your own lexicon. In Alexis Dimitriadis, Laura Siegel, Clarisa SurekClark, and Alexander Williams (eds.), University of Pennsylvania Working Papers in Linguistics 4, 201-225. Philadelphia: University of Pennsylvania.

Marantz, Alec. 2013. Locality domains for contextual allomorphy across the interfaces. In Ora Matushansky \& Alec Marantz (eds.), Distributed Morphology today: Morphemes for Morris Halle, 95-115. Cambridge, MA: MIT Press.

Merchant, Jason. 2015. How much context is enough? Two cases of span-conditioned stem allomorphy. Linguistic Inquiry 46(2). 273-303.

Partee, Barbara, Alice ter Meulen \& Robert E. Wall. 1993. Mathematical Methods in Linguistics. Dordrecht: Kluwer.

Picallo, M. Carme. 2008. Gender and number in Romance. Lingue e linguaggio 7(1). 47-66.

Sheehan, Michelle, \& Wolfram Hinzen. 2011. Moving towards the edge. Linguistic Analysis, 37(3-4), 405-458.

Veselinova, Ljuba (2016). Typology of Suppletion in Verb Paradigms. Amsterdam: John Benjamins.

Wiltschko, Martina. 2008. The syntax of non-inflectional plural marking. Natural Language \& Linguistic Theory 26(3). 639-694.

Wood, Jim. 2015. Icelandic Morphosyntax and Argument Structure. Dordrecht: Springer.

Wood, Jim \& Alec Marantz. 2017. The interpretation of external arguments. In Roberta D'Alessandro, Irene Franco \& Ángel J. Gallego (eds.), The verbal domain, 255-278. Oxford: Oxford University Press.

Zhang, Hong. 2007. Numeral Classifiers in Mandarin Chinese. Journal of East Asian Linguistics 16: 43-59.

Zhang, Niina Ning. 2012. Countability and Numeral Classifiers in Mandarin Chinese. In Diane Massam (ed.), Count and Mass Across Languages, 199-219. Oxford: Oxford University Press. 
